\title{
Minimum Required Distance of Strain Gauge from Specimen for Measuring Transmitted Signal in Split Hopkinson Pressure Bar Test
}

\author{
Daesung Kim ${ }^{1}$ and Hyunho Shin ${ }^{1, a}$ \\ ${ }^{1}$ Mechanics of Materials and Design Laboratory, Department of Materials Engineering, Gangneung-Wonju National University, Gangneung, \\ Gangwon-do 25457, Republic of Korea
}

\begin{abstract}
The minimum required distance of the strain gauge on the transmitted bar of the split Hopkinson bar has been determined from the position of a metallic specimen via an explicit finite element analysis. The minimum required distance was determined when the strain-time profiles at $r=0,0.5 R_{0}$ and $1.0 R_{0}$, were coincident $(r$ is the radial position and $R_{\mathrm{o}}$ is the radius of the bar.). The determined minimum required distance, $f(x)$, is presented as a function of the relative specimen diameter to that of the bar $\left(x=D / D_{0}\right): f(x)=-0.9385 x^{3}+0.6624 x^{2}-0.7459 x+$ $1.4478(0.1 \leq x \leq 0.9)$. This result demonstrates the Saint-Venant's principle of rapid dissipation of localized stress in transient loading. The result will be useful for the design/modification of the pseudo-one-dimensional impact instruments that utilise a stress pulse transmitted through the specimen. The result will also allow one to avoid unnecessarily remote strain gage position from the specimen.
\end{abstract}

\section{Introduction}

A strain rate-dependent constitutive equation $[1,2]$ is indispensable for the modelling and simulation-based design of solids and structures exposed to high strain rate events [3-6] such as crashes in high-speed transportation systems (airplanes, express trains, and automobiles), high-speed machining, blasting of rock and buildings, impact, penetration, and explosion. Stress-strain curves measured under the uniaxial stress condition at a wide range of strain rates are required for the calibration of a strain-rate-dependent constitutive equation. Pseudo-onedimensional impact systems such as the split Hopkinson bar (SHB) [7-11] and the direct impact bar (DIB) [1216] are widely used for measuring the uniaxial stressstrain curves of various materials at strain rates of approximately $10^{2}-10^{4} \mathrm{~s}^{-1}$ and over $10^{4} \mathrm{~s}^{-1}$, respectively.

In the DIB, the striker itself impacts the specimen directly and thus, there is no incident bar (Fig. 1(a)); the strain gauge is attached only to the transmitted bar. In the SHB (Fig. 1(b)), a disk-type specimen is sandwiched between the incident and transmitted bars; the strain gauges are attached to both the incident and transmitted bars (Fig. 1(b)). The strain gauge on the incident bar has to be sufficiently away (a distance of $x$ ) from the specimen $(x>L$, where $L$ is the striker length) to avoid the superposition of the incident and reflected pulses. For both the DIB and SHB, the strain gauge in the transmitted bar is essential for measuring the specimen stress.

In these instruments, the diameter of the specimen is usually smaller than that of the bar to avoid the radial expansion of the specimen beyond the bar diameter.

\footnotetext{
${ }^{\mathrm{a}}$ Corresponding author: hshin@gwnu.ac.kr
}

According to a recent study [10], the diameter of oxygenfree high thermal conductivity copper specimen needs to be far less than the bar diameter to obtain the stress-strain curve in the SHB test under a constant strain rate.
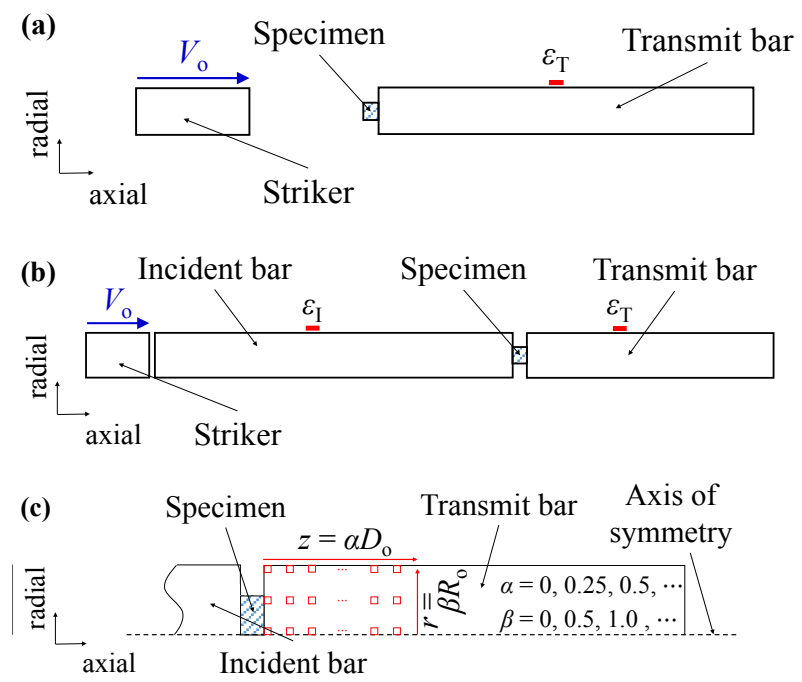

Figure 1. Schematics of (a) direct impact bar and (b) split Hopkinson pressure bar, and (c) considered gauge positions in this study $(z$ and $r$ are the axial and radial positions from the specimen and the axis of symmetry, respectively).

If the specimen diameter is smaller than that of the bar, stresses in the bar are not evenly distributed in the region near the specimen because a part of the bar end region is a free surface which is not in contact with the 
specimen. As a result, the measured stress on the bar surface near the specimen does not represent the bar stress in equilibrium with the specimen. Under such a circumstance, it is desirable to attach the strain gauge in the transmitted bar at a distance sufficiently remote from the specimen, where the stress (strain) is evenly distributed along the radial direction in the bar.

On the other hand, the shape of a stress pulse disperses as it travels the bar $[17,18]$, resulting in fluctuation in the measured curves of stress-strain and strain rate-strain using the SHB signals. The longer the distance of the strain gauge from the specimen in the transmitted bar, the higher the degree of dispersion, which necessitates dispersion correction to reduce fluctuation in the measured curves.

If the minimum required distance of the strain gage is revealed, it will deepen understanding of the stress wave propagation characteristics in the specimen-bar system, which is essential for the design and modification of the pseudo-one-dimensional impact instruments. Knowledge on minimum required distance will also allow one to avoid unnecessarily remote strain gage position from the specimen because an overly long distance will only increase the necessity of dispersion correction. In these regards, this study reveals the minimum required distance of the strain gauge on the transmitted bar that allows the measurement of the evenly distributed stress (strain) in the bar when the specimen diameter is smaller than that of the bar. In this work, compression is denoted with a positive sign.

\section{Numerical analysis}

A compression-mode SHB experiment was simulated using an explicit finite element analysis. The diameters $\left(D_{\mathrm{o}}\right)$ of the striker, incident bar, and transmitted bar were $20 \mathrm{~mm}$ while their lengths were $300,2,000$ and 1,000 mm, respectively. The length of the specimen was $5 \mathrm{~mm}$, while a range of specimen diameters were considered: $D / D_{\mathrm{o}}$ ratios were $0.10,0.25,0.5,0.75$ and 0.90 ( $D$ and $D_{\text {o }}$ are the initial diameters of the specimen and bar, respectively). The striker was initially $0.1 \mathrm{~mm}$ away from the bar, and its initial velocity was set as $15 \mathrm{~m} / \mathrm{s}$.

Considering the axial symmetry of the SHB system, half of the two-dimensional axisymmetric geometry was discretised using four-node bilinear axisymmetric quadrilateral elements (CAX4R). The sizes of the elements in the bar and specimen were approximately $1 \times$ 1 and $0.1 \times 0.1 \mathrm{~mm}^{2}$, respectively. The employed elements passed a separate mesh sensitivity test. No friction was considered between the contact surfaces.

Maraging steel (C300) and OFHC copper were considered as the materials for the specimen and bar (striker), respectively. The density, elastic modulus, and Poisson's ratio of maraging steel were considered to be $8,000 \mathrm{~kg} / \mathrm{m}^{3}, 190 \mathrm{GPa}$, and 0.29 , respectively. The corresponding values for OFHC copper were $8,940 \mathrm{~kg} / \mathrm{m}^{3}$, $115 \mathrm{GPa}$, and 0.31 , respectively. Plastic deformation behaviour of the OFHC copper specimen was described using the Johnson-Cook (JC) constitutive model [1]:

$$
\begin{gathered}
\sigma=\left(A+B \varepsilon^{n}\right)\left(1+C \ln \left(\dot{\varepsilon} / \dot{\varepsilon}_{\mathrm{o}}\right)\right)\left(1-T^{* \mathrm{~m}}\right) \\
T^{*}=\left(T-T_{\mathrm{ref}}\right) /\left(T_{\mathrm{m}}-T_{\mathrm{ref}}\right)
\end{gathered}
$$

where $\varepsilon, \dot{\varepsilon}$, and $T$ are the true plastic strain, true plastic strain rate, and temperature of the specimen, respectively; $A(90 \mathrm{MPa}), B(292 \mathrm{MPa}), n(0.31), C(0.025)$, and $m$ (1.09) are material parameters [1]; $\dot{\varepsilon}_{o}, T_{\text {ref }}$ are the reference values of the strain rate and temperature for calibration of the model; and $T_{\mathrm{m}}(1,356 \mathrm{~K})$ is the melting point of the specimen.

In the post processing stage, the profile of the axial strain in the incident bar $\left(\varepsilon_{\mathrm{l}}\right)$ was extracted from a surface element at $1,000 \mathrm{~mm}$ from the specimen. The profiles of the axial strain in the transmitted bar ( $\varepsilon_{\mathrm{T}}$ 's) were extracted from a series of elements illustrated in Fig. 1(c): the axial distances ( $z$ 's) of elements varied from zero to $1.5 D_{\mathrm{o}}$ at a step size of $0.25 D_{\mathrm{o}}$; three radial distances $(r$ 's) i.e., $r=0,0.5 R_{\mathrm{o}}$ and $1.0 R_{\mathrm{o}}$, were considered $\left(R_{\mathrm{o}}\right.$ is the radius of the bar).

The extracted signals in the numerical simulation were processed through a one-dimensional analysis [711]:

$$
\begin{aligned}
& \mathrm{s}=\left(A / A_{\mathrm{o}}\right) e_{\mathrm{T}}(t) \\
& \dot{e}=-2\left(C_{\mathrm{o}} / L\right) e_{\mathrm{R}}(t) \\
& e=-2\left(C_{\mathrm{o}} / L\right) \int_{0}^{t} e_{\mathrm{R}}(t) d t
\end{aligned}
$$

where, $s, \dot{e}$, and $e$ are the nominal stress, nominal strain rate, and nominal strain of the specimen, respectively; $A$ and $L$ are the initial cross-sectional area and initial length of the specimen, respectively; $e_{\mathrm{T}}$ and $e_{\mathrm{R}}$ are the nominal strain records of the transmitted and reflected pulses, respectively, and $t$ is time. $C_{\mathrm{o}}\left(=\left(E_{\mathrm{o}} / \rho_{\mathrm{o}}\right)^{1 / 2}\right)$ and $A_{\mathrm{o}}$ are the velocity of sound and cross-sectional area of the bar, respectively. The true stress-strain and true strain ratestrain curves of the specimen were determined from the corresponding engineering stress-strain and engineering strain rate-strain curves by assuming that the volume of the specimen remains constant during deformation.

To verify whether the strain signals extracted from a given axial position allows the reliable determination of the stress-strain and strain rate-strain curves, the strain rate equation in Ref. [10] was employed:

$$
\dot{\varepsilon}=\left(V_{\mathrm{o}}-2 A \sigma / A_{\mathrm{o}} \rho_{\mathrm{o}} C_{\mathrm{o}} \exp (-\varepsilon)\right) / L \exp (-\varepsilon)
$$

where $\varepsilon, \dot{\varepsilon}$, and $\sigma$ are true strain, true strain rate, and true stress of the specimen, respectively.

\section{Results and discussion}

\subsection{Determination of the gauge position}

The stress profiles at two different locations in the transmitted bar are presented in Fig. 2 for the case where the relative specimen diameter $\left(D / D_{\mathrm{o}}\right)$ is 0.5 . In Fig. 2(a), the magnitudes of stress pulses at $z=0.25 D_{\mathrm{o}}$ differ 
depending on the radial position. In Fig. 2(b), it can be seen that they coincide at $z=1.0 D_{\mathrm{o}}$
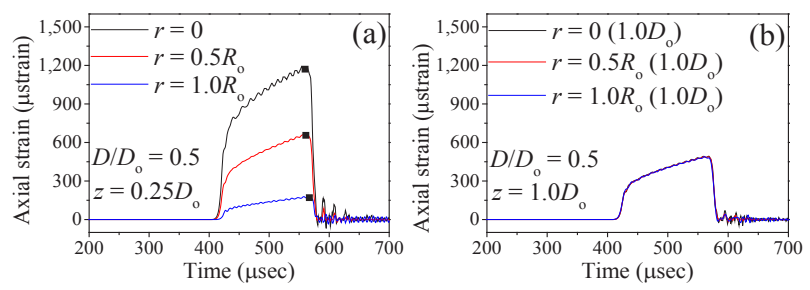

Figure 2. Axial stress profile at (a) $z=0.25 D_{\mathrm{o}}$ and (b) $1.0 D_{\mathrm{o}}$ for the case where $D / D_{0}$ ratio is 0.5 .

If the strain signals at $r=0,0.5$, and $1.0 R_{\mathrm{o}}$ coincide at a given axial distance $(z)$, the stress state in the bar can be regarded as being homogeneously distributed and thus the strain signal extracted at $r \geq R$ may be used to determine the specimen properties such as the dynamic stress-strain curve. To verify this point, the stress-strain and strain rate-strain curves were constructed using strain signals, i.e., $\varepsilon_{\mathrm{R}}$ and $\varepsilon_{\mathrm{T}}\left(z=1.0 D_{\mathrm{o}} ; r=1.0 R_{\mathrm{o}}\right)$ for the case where $D / D_{\mathrm{o}}=0.5$. The result is presented in Fig. 3, where the curves $S$ and $R$ were determined via Eqs. (1)-(3).
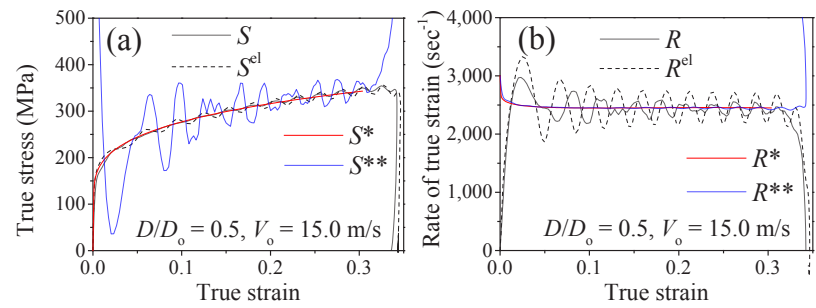

Figure 3. Curves of (a) stress-strain and (b) strain rate-strain determined for the case where $D / D_{0}=0.5$.

Included in Fig. 3 are curves $S^{\mathrm{el}}$ and $R^{\mathrm{el}}$, which were determined by averaging the values of the axial stress and strain rate of the specimen elements. Curves $S^{\mathrm{el}}$ and $R^{\mathrm{el}}$ are available only in simulation (not in experiment).

Fig. 3 also includes curves $R^{*}$ and $S^{*}$, which were obtained by simultaneously solving the constitutive equation of the specimen (Eq. (1)) and the strain rate equation (Eq. (5)) using the Excel program available in Ref. [10]. Curves $S^{*}$ and $R^{*}$ are the stress-strain and strain rate-strain curves predicted based the theory of specimen strain rate in the $\mathrm{SHB}^{10}$ and the constitutive equation. In the experiment, the constitutive parameters of the JC model can be reasonably estimated from quasistatic tests via the method described in Ref. [10]. Curves $S^{*}$ and $R^{*}$ determined in this way can be referred to before carrying out the SHB experiment.

Finally, Fig. 3 includes curves $S^{* *}$ and $R^{* *}$, which were obtained by applying curves $S$ and $R$, respectively, to Eq. (5). Consistency of curves $S^{* *}$ and $R^{* *}$ with $S$ and $R$, respectively, can be used to verify the reliability of the experimental curves of $S$ and $R$, respectively.

It can be observed from Fig. 3(a) that the curves $S, S^{\mathrm{el}}$, $S^{*}$ and $S^{* *}$ are consistent. In Fig. 3(b), the curves $R, R^{\mathrm{e}}$, $R^{*}$, and $R^{* *}$ are also consistent. From these observations, it can be confirmed that the strain signals $\varepsilon_{\mathrm{I}}$ and especially $\varepsilon_{\mathrm{T}}$ at $z=1.0 D_{\mathrm{o}}$ is reliable, where $\varepsilon_{\mathrm{T}}$ at $r=0$, $0.5 R_{\mathrm{o}}$ and $1.0 R_{\mathrm{o}}$ coincide. Therefore, this study determines the strain gauge position $\left(z_{\mathrm{c}}\right)$ on the transmitted bar when $\varepsilon_{\mathrm{T}}(z ; r=0), \varepsilon_{\mathrm{T}}\left(z ; r=0.5 R_{\mathrm{o}}\right)$, and $\varepsilon_{\mathrm{T}}\left(z ; r=1.0 R_{\mathrm{o}}\right)$, coincide.

\subsection{Required strain gauge distance}

In Fig. 2(a), the maximum strain value at a given pulse profile is marked as the closed square. This maximum value of a given profile was normalized to the corresponding value of the profile at $z=z_{\mathrm{c}}$, and the normalized values $\left(\varepsilon_{\mathrm{T}}(z) / \varepsilon_{\mathrm{T}}\left(z_{\mathrm{c}}\right)\right)$ at $r=0$ are presented in Fig. 4(a) for a range of the relative specimen diameters $\left(D / D_{\mathrm{o}}\right.$ 's $)$. The normalized values at $r=0.5 R_{\mathrm{o}}$ and $1.0 R_{\mathrm{o}}$ are also presented in Figs. 4(b) and 4(c), respectively.

At $r=0$ (Fig. 4(a)) and $0.5 R_{\mathrm{o}}$ (Fig. 4(b)), interestingly, the normalized value of the axial strain is maximized at the axial position between zero and $z_{\mathrm{c}}$. This maximization of the axial strain is similar to the phenomenon observed in a quasi-static indentation where the maximum axial stress does not appear at the contact surface but at a distance slightly below the contact surface $[19,20]$.
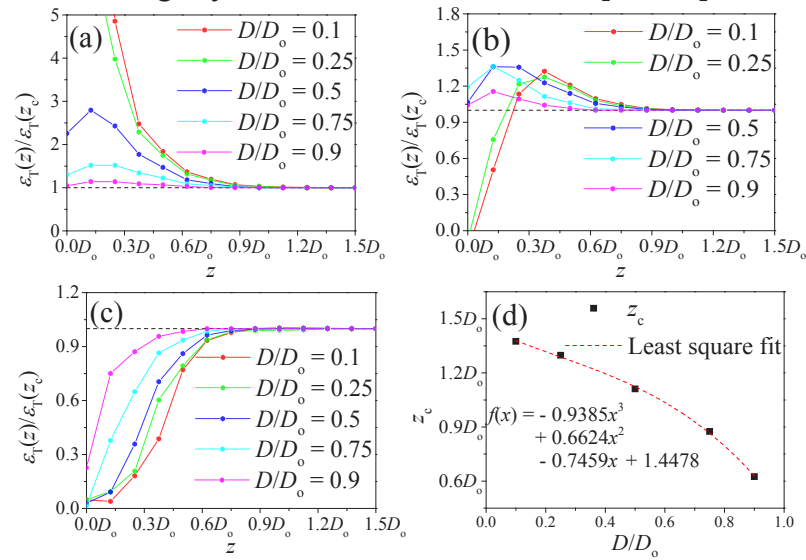

Figure 4. Normalized values $\left(\varepsilon \mathrm{T}(z) / \varepsilon \mathrm{T}\left(z_{\mathrm{c}}\right)\right)$ at (a) $r=0$, (b) $r=$ $0.5 R_{\mathrm{o}}$, and (c) $r=1.0 R_{\mathrm{o}}$ for a range of $D / D_{\mathrm{o}}$ ratios. (d) Minimum required distance $\left(z_{\mathrm{c}}\right)$ for the gauge position in the transmitted bar.

At $r=1.0 R_{\mathrm{o}}$ (Fig. 4(c)), the normalized strain value is generally lower at the axial position $(z)$ near the specimen; the smaller the specimen diameter, the lower the strain value. This observation results from the fact that, when the specimen diameter is smaller than the bar, the stress is not equally distributed in the transmitted bar as a part of the bar end is in contact with the free surface instead of the specimen.

As mentioned, the strain gauge positions $\left(z_{\mathrm{c}}\right.$ 's) in the transmitted bar were determined when $\varepsilon_{\mathrm{T}}(z ; r=0), \varepsilon_{\mathrm{T}}(z ; r$ $\left.=0.5 R_{\mathrm{o}}\right)$, and $\varepsilon_{\mathrm{T}}\left(z ; r=1.0 R_{\mathrm{o}}\right)$, coincide. The determined result for a range of relative specimen diameters $\left(D / D_{\mathrm{o}}\right.$ ratios) is shown in Fig. 4(d). As can be seen in Fig. 4(d), the minimum required distance increases as the specimen diameter decreases. Included in Fig $4(\mathrm{~d})$ is the least square fit using a third order polynomial: $f(x)=-$ $0.9385 x^{3}+0.6624 x^{2}-0.7459 x+1.4478(0.1 \leq x \leq 0.9)$, where $f(x)$ is the minimum required distance and $x=D / D_{0}$. 
This curve can be referred to for determining the minimum distance for positioning strain gauge on the transmitted bar for specimens with a different diameter from the ones used for numerical analysis in this study.

\subsection{Verification for extreme cases}

The minimum required distance shown in Fig. 4(d) was obtained for the OFHC specimen with a given plastic property described by the parameters $A, B, n, C$, and $m$ in Eq. (1). The parameter $A$ determines the yield strength of the material. To verify whether the determined distance indicated in Fig. 4(d) can be used for a higher-strength material, this study additionally considered a hypothetical specimen with an $A$ value of $800 \mathrm{MPa}$ (the rest of the JC parameters are the same as the OFHC copper specimen). Example cases of the strain profiles when $D / D_{\mathrm{o}}=0.5$ at $z$ $=1.0 D_{\mathrm{o}}$ are shown in Fig. 5 (a).
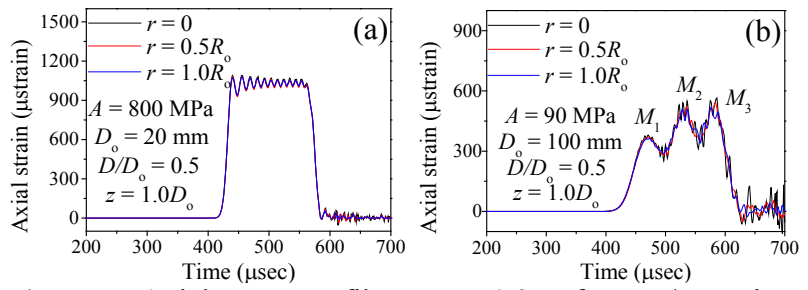

Figure 5. Axial stress profiles at $z=1.0 D_{\mathrm{o}}$ for a $D / D_{\mathrm{o}}$ ratio of 0.5 . The yield strength of the specimen and the bar diameter $\left(D_{\mathrm{o}}\right)$ were (a) $800 \mathrm{MPa}$ and $20 \mathrm{~mm}$, respectively, and (b) 90 $\mathrm{MPa}$ and $100 \mathrm{~mm}$, respectively.

In Fig. 5(a), magnitudes of the $\varepsilon_{\mathrm{T}}$ profiles at $r=0,0.5$ and $1.0 R_{\mathrm{o}}$ reasonably coincide, which is consistent with the result in Fig. 4(d) where $z_{\mathrm{c}}=1.0 D_{\mathrm{o}}$ when $D / D_{\mathrm{o}}=0.5$ for the OFHC specimen with an $A$ value of $90 \mathrm{MPa}$. Therefore, the quantified minimum distance in Fig. 4(d) can be used for specimens with a yield strength of at least up to $800 \mathrm{MPa}$.

The minimum required distance shown in Fig. 4(d) was obtained by considering a bar diameter of $20 \mathrm{~mm}$. To verify whether the determined result in Fig. 4(c) can be used for a bar with a larger diameter, this study additionally considered the case where the diameters of the specimen and bar were enlarged while their axial lengths were not varied. Example cases of the strain profiles when $D / D_{\mathrm{o}}=0.5$ at $z=1.0 D_{\mathrm{o}}$ are shown in Fig. 5 (b) for the case when $D_{\mathrm{o}}$ was $100 \mathrm{~mm}$.

In Fig. 5(b), there are only three peaks in the strain pulse period (marked as $M_{1}, M_{2}$, and $M_{3}$ ). In our separate analysis, number of such peaks decreases with the bar diameter (not shown), which results from the deviation of the impact system from the one-dimensional nature with the increase of the bar diameter. The issue on the allowable maximum bar diameter for the reliable measurement of specimen properties needs further study for clarification. From the observation that the three curves at $r=0,0.5 R$, and $1.0 R$ in Fig. 5(b) are reasonably coincident, the quantified result in Fig. 4(d) can be used for a bar diameter of up to at least $100 \mathrm{~mm}$. In the literature [21], the necessity of correction for the strain signal measured on the bar surface to obtain the signal at the bar axis was reported even for the case where the strain gage was sufficiently remote from the specimen. Further detailed numerical verification is necessary to reaveal a more accurate maximum bar diameter, up to which there is no need for the surface signal correction to obtain the bar axis signal.

\subsection{Saint-Venant's principle}

Saint-Venant's principle [22] states that "If the forces acting on a small portion of the surface of an elastic body are replaced by another statically equivalent system of forces acting on the same portion of the surface, this redistribution of loading produces substantial changes in the stresses locally, but has a negligible effect on the stresses at distances which are large in comparison with the linear dimensions of the surface on which the forces are changed". An example of the principle is schematically illustrated in Fig. 6, where the same magnitude of load (100 kgf) is applied to the bar from a small-diameter (high-density) or a large-diameter (lowdensity) object. Although the local stress fields near the objects are different for the two comparing cases, they are practically the same after a short distance from the bar/object contact surface. In this regard, the principle is conveniently called 'Saint-Venant's principle of rapid dissipation of localized stress' [23]. The principle was generally considered in quasi-static loading. The result presented in this study, Figure 4(d), has a meaning in that it serves as an example of the Saint-Venant's principle in transient loading. Figure 4(d) describes the principle on a quantitative base, which supplements the qualitative description of the principle in recent studies [24, 25].

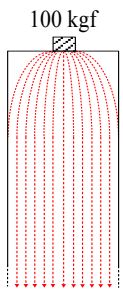

(a)

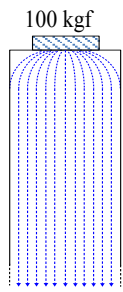

(b)

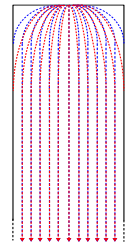

(c)
Figure 6. Force lines when a quasi-static load (100 kgf) is applied to the bar from (a) a small-diameter or (b) a largediameter disk. (c) Comparison of the force lines.

\section{Conclusions}

Numerical analyses of the split Hopkinson bar experiment were carried out via an explicit finite element analysis for a range of relative specimen diameters to that of the bar $\left(D / D_{\mathrm{o}}\right)$. Based on the result of numerical analyses, the minimally required distance of the strain gauge from the specimen surface in the transmitted bar was quantified from the viewpoint of the coincidence of the strain profiles at $r=0,0.5 R_{\mathrm{o}}$ and $1.0 R_{\mathrm{o}}$. The obtained result is $f(x)=-0.9385 x^{3}+0.6624 x^{2}-0.7459 x+1.4478$ $(0.1 \leq x \leq 0.9)$, where $f(x)$ is the minimum required distance and $x=D / D_{0}$. This result can be used for specimens with a yield strength of at least up to $800 \mathrm{MPa}$ and for a bar diameter of up to at least $100 \mathrm{~mm}$. The 
minimum required distance is an example of the SaintVenant's principle of rapid dissipation of localized stress in transient loading. The result will be useful for the design and modification of the pseudo-one-dimensional impact instruments that utilise a stress pulse transmitted through the specimen. The result will also allow one to avoid unnecessarily remote strain gage position from the specimen because an overly long distance will only increase the necessity of dispersion correction.

\section{Acknowledgement}

This study was financially supported by a National Research Foundation of Korea grant under contract No. 2019R1H1A2039686, funded by the Ministry of Science and Technology (Korea).

\section{References}

1. G.R. Johnson, W.H. Cook, A Constitutive Model and Data for Metals Subjected to Large Strains, High Strain Rates and High Temperatures. In: Proc. 7th Int symp ballistics pp. 541-547 (The Hague, Netherlands, 19-21 April 1983).

2. H. Shin, J.-B. Kim, A Phenomenological Constitutive Equation to Describe Various Flow Stress Behaviors of Materials in Wide Strain Rate and Temperature Regimes, J. Eng. Mater. Technol. 132, 021009 (2010).

3. Y.-H. Yoo, S.-H. Paik, J.-B. Kim, H. Shin, Performance of a Flying Cross Bar to Incapacitate a Long-Rod Penetrator Based on a Finite Element Model, Eng. Comput., 29(4), 409-415 (2013).

4. D.-H. Kim, H. Shin, D. Jung, A Numerical Study on Jet Formation and Penetration Characteristics of the Shaped Charge with an Aspect Ratio of 2.73 and a High-Strength Copper Liner (in Korean), Kor. J. Comput. Design Eng., 24(1), 73-84 (2019).

5. D. Kim, J.-H. Lee, H. Shin et al., A Numerical Study on the Influence of the Flow Stress of Copper Liner on the Penetration Performance of a Small-Caliber High Explosive (in Korean), Kor. J. Comput. Design Eng., 24(4), 300-310 (2019).

6. H. Shin H, H.-J. Lee, Y.-H. Yoo, W. Lee, A Determination Procedure for Element Elimination Criterion in Finite Element Analysis of High-StrainRate Impact/Penetration Phenomena, JSME Int. J. 47(1), 35-41 (2004).

7. H. Kolsky, An investigation of the Mechanical Properties of Materials at Very High Rates of Loading. Proc. Phys. Soc. Lond. Sect. B, 62(11), 676-700 (1949).

8. M.A. Meyers, Dynamic behavior of materials (John Wiley \& Sons, Inc, New York, 1994).

9. M.M. Al-Mousawi, S.R. Reid, W.F. Deans, The Use of the Split Hopkinson Pressure Bar Techniques in High Strain Rate Materials Testing, Proc. IMechE, Part C: J. Mech. Eng. Sci., 211, 273-292 (1997).

10. H. Shin, J.-B. Kim, Evolution of Specimen Strain Rate in Split Hopkinson Bar Test, Proc. IMechE,
Part C: J. Mech. Eng. Sci., 233(13), 4667-4687 (2019).

11. H. Shin, J.-B. Kim, Understanding the Anomalously Long Duration Time of the Transmitted Pulse from a Soft Specimen in a Kolsky Bar Experiment, Int. J. Precision Eng. Manufact., 17(2), 203-208 (2016).

12. H. Couque, The Use of the Direct Impact Hopkinson Pressure Bar Technique to Describe Thermally Activated and Viscous Regimes of Metallic Materials, Phil. Trans. R. Soc. A, 372, 20130218 (2013).

13. L.J. Lea, A.P. Jardine, Application of Photon Doppler Velocimetry to Direct Impact Hopkinson Pressure Bars, Rev. Sci. Instruments 87, 023101 (2016).

14. R. Othman, S. Aloui, A. Poitou, Identification of Non-Homogeneous Stress Fields in Dynamic Experiments with a Non-Parametric Method, Polym. Testing 29, 616-623 (2010).

15. J.Z. Malinowski, J.R. Klepaczko, Z.L. Kowalewski, Miniaturized Compression Test at Very High Strain Rates by Direct Impact, Exp. Mech., 47, 451-463 (2007).

16. F. Kamler, P. Niessen, R.J. Pick, Measurement of the Behaviour of High Purity Copper at Very High Rates of Straining, Canad. J. Phys., 73(5-6), pp. 295-303 (1995).

17. Gorham DA. A Numerical Method for the Correction of Dispersion in Pressure Bar Signals. J. Phys. E: Sci. Instum., 16, 477-479 (1983).

18. Z. Li, J. Lambros, Determination of the Dynamic Response of Brittle Composites by the Use of the Split Hopkinson Pressure Bar, Compos. Sci. Tech., 59, 1097-1107 (1999).

19. Y.-H. Yoo, W. Lee, H. Shin, Spherical NanoIndentation of a Hard Thin Film/Soft Substrate Layered System: II. Evolution of Stress and Strain Fields, Modelling Simul. Mater. Sci. Eng., 12, 69-78 (2004).

20. R.A.C. Slater, Engineering Plasticity, Theory and Application to Metal Forming Processes (Macmillan, London, 1977).

21. A. Tyas and A.J. Watson, An Investigation of Frequency Domain Dispersion Correction for Pressure Bar Signals, Int. J. lmpact Eng., 25, 87-101 (2001).

22. B. De Saint-Venant, Mémoire sur la Torsion des Prismes (Memory on the Torsion of the Prisms), Mém. des Savants étrangers, 14, 233-560 (1855).

23. Punmia BC, Jain AK and Jain AK. Mechanics of Materials. (Laxmi Publications (P), Ltd., New Delhi 2001).

24. H. Shin, D. Kim, One Dimensional Analyses of Striker Impact on Bar with Different General Impedance, Proc. IMechE, Part C: J. Mech. Eng. Sci., https://doi.org/10.1177/0954406219877210 (2019).

25. H. Shin, J.-H. Lee, J.-B. Kim, S.-I. Sohn, Design Guidelines for the Striker and Transfer Flange of a Split Hopkinson Tension Bar and the Origin of Spurious Waves, Proc. IMechE, Part C: J. Mech. Eng. Sci., https://doi.org/10.1177/0954406219869984 (2019). 